\title{
LA PRÁCTICA PROFESIONAL COMO PROBLEMA DE APRENDIZAJE: ORIENTACIONES PARA LA APLICACIÓN DE UN MODELO DE DESARROLLO DE COMPETENCIAS
}

\author{
Marisa Guzmán Munita
}

\begin{abstract}
RESUMEN
Este artículo es un avance de una investigación en curso, cuyo objetivo es conocer el impacto de un modelo pedagógico de desarrollo de competencias. La muestra la compone un grupo de estudiantes de pedagogía en castellano, en práctica profesional final. El modelo provee una serie de herramientas orientadas a que el futuro profesor de aula desarrolle competencias profesionales que estimulen su perfeccionamiento social y personal, de manera de favorecer que el conjunto de conocimientos, habilidades y actitudes que el currículum propone y que él administra, propicien el entendimiento fundamental entre los sujetos y la elaboración de soluciones a problemas prácticos, que aporten a la labor conjunta de construir una sociedad más humana y equitativa.
\end{abstract}

Palabras clave: modelo pedagógico, pedagogía en castellano, práctica profesional, competencias profesionales

\section{PROFESSIONAL PRACTICE AS A LEARNING DILEMMA: GUIDELINES FOR APPLYING A MODEL OF COMPETENCE DEVELOPMENT}

\begin{abstract}
This article is a preview of an ongoing investigation aimed at determining the impact of a pedagogical model for competences development. The sample comprised a group of Spanish language teacher training students, in their final practicum component. The model provides a set of tools for the future classroom teachers to develop professional competences that foster future teachers' social and personal development with a view to accommodate a large body of knowledge, skills and attitudes. So this process can nurture the fundamental understanding between the individuals and the solutions to practical problems that contribute to the collaborative efforts to build a more humane and equitable society.
\end{abstract}

Keywords: Teaching model, Spanish Language Teacher Training Program, Professional Practice, Professional Skills.

* Licenciada en Comunicación Social, Magíster en Lingüística. Académica de la Facultad de Educación en Humanidades y Ciencias, Universidad Católica Silva Henríquez. Coordinadora de prácticas profesionales de Castellano (UCSH). Docente de post grado Universidad Nacional Andrés Bello. Doctoranda de Ciencias Pedagógicas, La Habana, Cuba. Correo electrónico: journalistmgm@gmail.com 


\section{Introduccción}

En la Universidad Católica Silva Henríquez (UCSH) se enmarca el estudio que revisa cómo fortalecer el proceso de formación docente, y se enfoca específicamente en estudiantes de pedagogía en castellano -futuros profesores de Lengua castellana y comunicación- una cohorte de 54 estudiantes en práctica profesional. ${ }^{I}$ Esta selección se hace sobre la base de considerar que este sector de aprendizaje proporciona el cimiento a través del cual las demás áreas del saber se desarrollan. Como se declara en su enfoque curricular: “...constituye en sí mismo, una herramienta fundamental para la integración de las personas en una sociedad moderna, participativa y democrática [...] propicia el desarrollo del pensamiento crítico y reflexivo, la creatividad y el diálogo.” (MINEDUC 2009, p. I).

A partir de la implementación del proyecto de mejoramiento de la calidad y equidad de la educación (MECESUP 2009-201 I, De Noguera et al. 2009), la UCSH reivindica la relevancia de la práctica profesional, definiéndola como el espacio que organiza las competencias disciplinares y profesionales esenciales para el desarrollo del ejercicio docente, y la enmarca a través de una rúbrica de competencias, con la que evalúa la preparación de sus estudiantes para asumir la labor de enseñar.

Durante la realización de la práctica profesional, se inserta a los practicantes en comunidades educativas auténticas, siendo el aula el espacio donde se verifica el desarrollo de las competencias manifiestas en el modelo de formación, el perfil de egreso y lo que establecen los diversos programas de castellano, proyectando: "Promover la formación de profesionales con principios y valores, conocimientos y competencias que les permitan un efectivo desempeño laboral y la integración responsable en el desarrollo de una sociedad justa, democrática y solidaria" (Modelo de formación de la Universidad Católica Silva Henríquez 2003, p. I I).

I Investigación que se inscribe en el contexto de la tesis doctoral de la autora, en la Universidad de Ciencias Pedagógicas “Enrique José Varona”. La Habana, Cuba. 
Sin embargo, la dispar condición con que los estudiantes llegan a asumir su práctica profesional en su último año de formación, dificulta el objetivo conjunto de que estos nuevos profesores respondan a los diversos requerimientos disciplinares que impone la práctica y tomen decisiones con fundamentos científicos; lo que genera una contradicción en torno al deber ser, y lo que ocurre en el aula (Cox 1999; Informe Delors 1996).

Esto dio lugar a una investigación que persigue orientar el proceso formativo final de los practicantes, a fin de contribuir a elevar las competencias de aquellos que necesiten mayor apoyo y compromete la adherencia de los profesores supervisores para implementarlo.

La naturaleza de las debilidades con que llegan los estudiantes a esta instancia, es diversa: débil formación en aspectos disciplinares (lo que les impide exponer con fluidez ciertas temáticas), escasa conciencia lingüística (lo que les dificulta la selección/elaboración de material de apoyo y actividades que consideren el contexto sociocultural de los estudiantes), y otros derivados del desconocimiento de una estructura que los oriente en el trabajo de aula. Diversas investigaciones sostienen la pertinencia de desarrollar habilidades que permitan avanzar hacia el perfeccionamiento de las competencias genéricas y específicas que requiere el conocimiento a fin de transformarlo, lo que demanda propiciar el desarrollo del pensamiento humano que permita comprender, interpretar y valorar los fenómenos críticamente, a fin de prepara a los sujetos para la vida (Álvarez de Zayas 1999; Albornoz 200I; Aebli 200I).

En este escenario, se justifica la pertinencia de elaborar un modelo que no sólo potencie el desarrollo de las competencias que son evaluadas, sino lo que denominamos 'competencias profesionales' que enmarcamos en: competencias didácticas, comunicativas e investigativas, cuyo desarrollo propicia la transformación del conocimiento, la resolución de variados problemas emanados del ejercicio de la profesión y la utilización de métodos científicos y tecnológicos para describir, explicar y predecir la realidad, en 
concordancia con hacer de los educandos y sus circunstancias, objeto permanente de investigación científica (Martínez Quijano 2008).

\section{El sistema de acompañamiento de la UCSH}

El documento oficial que orienta la labor de los profesoressupervisores, señala que éstos deben facilitar en los estudiantes: “... el desarrollo de competencias técnicas (poniendo énfasis en las metodologías utilizadas por éstos); competencia clínica (es decir habilidades de razonamiento y resolución de problemas prácticos); competencias personales (intra e interpersonales); competencia crítica (relacionada con el reconocimiento de temas sociales, éticos y morales, así como la reflexión sobre el proceso en su totalidad)" lo que implica apoyarlos con diversos recursos. (Cuaderno de trabajo de práctica profesional. 200I, p. 6). No obstante, en la experiencia, el profesor supervisor no cuenta con orientaciones específicas respecto de cómo conducir el proceso de desarrollo de las competencias perseguidas. Las estrategias que utiliza cada quien para dar cuerpo al objetivo conjunto de apoyarlas, no ha sido sistematizado. Cada supervisor aplica su criterio para interpretar estas demandas, y en base a su mayor o menor experiencia, provee y comparte recursos metodológicos y didácticos.

Esto ha dado lugar a que el acompañamiento sea un espacio que se conciba de maneras muy diversas. Algunos asumen tácitamente que dicho acompañamiento supone dar apoyo concreto para nivelar las competencias que se aprecien disminuidas. En cambio otros, consideran que no es labor de los supervisores retomar aspectos que se supone fueron adquiridos por los practicantes, tras haber aprobado los diversos cursos de formación. Por su parte, el procedimiento institucional demanda tres instancias evaluativas: un portafolio virtual, que a través de una rúbrica de desempeño, objetiva las competencias asociadas al Acto Docente, desarrollada en proceso con el profesor supervisor, al que se le entregan evidencias escritas, gráficas 
y audiovisuales, mediante envíos semanales al aula virtual. ${ }^{2}$ En cada reunión semanal, el supervisor retroalimenta los trabajos realizados y finalmente, evalúa ese aspecto del desempeño del estudiante, con un $30 \%$ de ponderación.

La segunda evaluación se corresponde con la observación de aula que también realiza el profesor-supervisor (30\%), la que supone la demostración del máximo desempeño del practicante, por cuanto se programa de común acuerdo. Esta pauta de observación contempla cuatro áreas fundamentales a observar:

- Ámbito de la clase

- Estilo metodológico del Alumno en Práctica

- Desarrollo de la clase en general

- Aspectos propios de la especialidad

Esta observación permite apreciar ciertas características propias del practicante, en las que debe evidenciarse como comunicador eficiente, haciendo uso de un amplio repertorio léxico, dinámico, con una metodología adecuada al contexto, competente en su manejo conceptual, grupal y de conflictos, con conocimientos de didáctica y uso de TIC. Esta observación se realiza en función de una pauta sugerida por la universidad, la que los supervisores tienen libertad de complementar con observaciones y sugerencias. Esta instancia, también es retroalimentada por los supervisores en las reuniones semanales.

La tercera, la constituye la pauta para elaboración de portafolio con que el profesor colaborador objetiva el desempeño del practicante, y es la que tiene mayor ponderación (un 40\% o 50\%, según se trate de Práctica I o II). Esto, en consideración a que a este profesor le asiste la labor de guiar el desarrollo del practicante en el establecimiento, por ser quien vivencia los aciertos y desaciertos del proceso del estudiante a su cargo. Por lo anterior, se le suman a la competencia del Acto Docente, evaluada por el supervisor, otras tres: Fundamentos de la Enseñanza, Relación con el Medio Escolar y Desarrollo e Identidad Profesional.

2 Se utiliza la plataforma moodle. 
Ante estas tres instancias evaluativas, el conjunto de evidencias seleccionadas y elaboradas por el practicante, deben demostrar su solidez disciplinar y revelar su conciencia lingüística, competencias sociales y comunicativas, tan necesarias e inaplazables para contribuir a que el futuro profesor especialista, no sólo maneje saber conceptual, sino que sea capaz de transferirlo (Verret 1975; Zabalza 2003). Lo último pone en juego un conjunto de habilidades didácticas que lo evidencien como eficiente comunicador, con manejo de un discurso oral acorde al rango de profesor, que se desarrolle en una estructura clara, coherente al contexto y a los momentos de la interacción comunicativa.

\section{El instrumento de evaluación}

El portafolio es una instancia que permite apreciar variadas competencias asociadas al ejercicio profesional: lo que deben saber y deben poder bacer, quienes se insertan al mercado laboral. El escenario educativo actual demanda un profesional que se muestre eficiente, flexible, innovador y creativo. Y para la consecución de este desafío, el sistema de evaluación de práctica se levanta como un espacio que permite la interacción continua, la construcción en proceso y la retroalimentación entre docente y discente.

La UCSH proyecta que sus futuros profesores de castellano, posean "dominio eficiente de las competencias profesionales y/o disciplinares” (Cuaderno de práctica profesional, Op. Cit. p. 3), las que describe de la siguiente manera:

- Comprender y analizar saberes, fenómenos y procesos desde diferentes perspectivas y marcos de referencia: implica integrar saberes, procedimientos y actitudes, a fin de responder a las demandas de los diversos episodios que se presenten en la praxis misma. Del mismo modo, implica la cualificación crítica de los distintos procesos y su mediación, lo que compromete el ejercicio de competencias investigativas para ampliar los marcos referenciales desde los que emane el análisis. 
- Resignificar como propio, el saber teórico y práctico de la disciplina, desarrollando su capacidad analítica, de razonamiento lógico: compromete demostrar flexibilidad, para asumir que ser profesor demanda actualizar permanentemente los conocimientos disciplinares y autocrítica para reflexionar éticamente sobre el propio quehacer. Esto se verifica en la interacción áulica, donde la apropiación de estas competencias debieran lograr que el profesor integre instancias como el diálogo y trabajo cooperativo, en los que comparta su saber, pero también reconozca los aportes de otros (personas o disciplinas). En este cometido, resultan relevantes las competencias comunicativas, en términos orales, escritos, simbólicos y mediáticos.

- Manifestar autonomía en el ejercicio de su profesión: De modo de tomar decisiones pertinentes y ajustadas a las distintas situaciones que enfrente o en las que se desempeñe.

- Reconocer la dimensión social y trascendente de la profesión: Reconociendo que el rol del profesor implica la responsabilidad de educar en valores y respeto por la dignidad de todas las personas, desarrollando la socialización de los individuos, el fortalecimiento de la familia y la conciencia cívica, a fin de contribuir a forjar una sociedad más justa, democrática, solidaria y pluralista, con una opción claramente centrada en los problemas propios de los sectores sociales más desposeídos.

- Respetar en su vida personal y profesional los rasgos característicos de la institución: En el contexto de una universidad católica y salesiana, la escala de valores comprometida en el ejercicio de la profesión debiera ser concordante con una visión humanista y cristiana de la persona, concebida como un ser trascendente, digno de respeto, desde su concepción. ${ }^{3}$

De esta forma, este posicionamiento queda de manifiesto tanto en el portafolio virtual, como en la observación auténtica, cuyos resultados dan cuenta de la calidad de profesionales que estamos formando.

3 Parafraseado de Op. Cit. p. II-I2. 


\section{El estudio}

La presente investigación aspira a dar respuesta a ciertos vacíos apreciados en el sistema de acompañamiento con que la UCSH enmarca el proceso de formación final, de futuros profesores de Castellano. Si bien se reconoce que la elaboración eficiente de evidencias de desempeño a lo largo de la práctica profesional, compromete las competencias de entrada de cada estudiante -algo en que los profesores supervisores no tienen ingerencia-, sí compete reflexionar sobre el trabajo de acompañamiento que se está realizando, para establecer elementos de apoyo para aquellos estudiantes que, tras la primera evaluación de proceso, demostraron desempeños inconsistentes.

Con todo, el sistema de acompañamiento que provee la UCSH destaca por la forma en que ha concebido y estructurado este proceso. Se enfatiza el enorme acierto que implica propiciar el desarrollo de competencias integrales, en la búsqueda por modernizar el sistema de evaluación de prácticas. Así, por ejemplo, el portafolio que provee la institución, nace del trabajo de connotados investigadores (Noguera y otros 2010) y persigue ofrecer una propuesta formativa de calidad, que permita acreditar la consecución de competencias afines al perfil profesional que promueve la universidad. En oposición al tradicional sistema de evaluación para esta instancia -el Informe meramente descriptivo-, el portafolio articula el desempeño del futuro docente en relación al conjunto de competencias que persigue acreditar. Estas competencias permiten apreciar el capital disciplinar, metodológico y didáctico con que nuestros estudiantes se posicionan en los diversos establecimientos a los que son asignados. Se ha señalado que uno de los temas fuertemente discutidos en las actuales investigaciones pedagógicas, no escatiman esfuerzos en abordar los diversos aspectos asociados tanto a saber la disciplina, como a saber enseñarla (Frade 2006; Pérez Gómez 2007; Aldape 2008; Gimeno Sacristán 2008). En este contexto, resulta pertinente proyectar orientar la formación de los futuros profesores, de manera de propiciar el manejo de diversas estrategias que les permitan desenvolverse con fluidez en su praxis. 
Son precisamente las condiciones en que se da el proceso de formación final, se pretende fortalecer el modelo que presenta este estudio. Por ser este el espacio donde confluyen los profesores supervisores y alumnos en su más diversa y heterogénea condición, el modelo anhela aunar criterios metodológicos y didácticos, para lo que provee orientaciones que buscan hacer más perfectible el proceso. Se aspira a afianzar aquellas habilidades que lleven a una práctica áulica competente, a través de la cual se podría objetivar la consecución de los diversos estándares que vaya imponiendo el sistema educativo.

Para efectos de esta investigación, se tomaron como base los resultados de la observación de aula y del portafolio virtual, del primer semestre 20I I. El universo lo constituyó la cohorte conformada por 54 practicantes de este período, de los cuales 44 correspondieron al nivel 900 (Práctica I) y I0 al nivel I000 (Práctica II).

La muestra la conformó el conjunto de estudiantes que demostraron resultados que los dejaron en el rango de básicos e insuficientes ${ }^{4}$, en alguna de las cuatro competencias evaluadas en el Acto Docente y/o sus 16 indicadores de desempeño, lo que se complementó con la observación de aula. Ambas instancias evaluativas sirvieron para establecer el diagnóstico inicial.

Resulta interesante señalar que la rúbrica del portafolio, formula las competencias generales y específicas asociadas al Acto Docente de la siguiente manera:

4 Existiendo cuatro rangos: insuficiente, básico, competente y destacado. 
La práctica profesional como problema de aprendizaje: orientaciones para la aplicación de un modelo de desarrollo de competencias - Marisa Guzmán Munita

Figura I: Tomada del Portafolio de Prácticas Profesionales (20II, p. 9).

\begin{tabular}{|c|c|c|}
\hline Baver & Coneterada & Mikaler \\
\hline \multirow{17}{*}{$\begin{array}{l}\text { Acto } \\
\text { oocenefe }\end{array}$} & \multirow{5}{*}{ 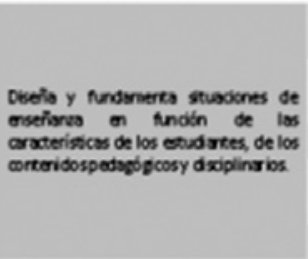 } & 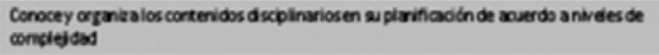 \\
\hline & & 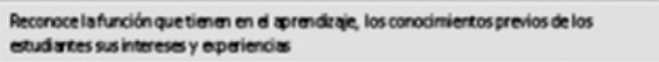 \\
\hline & & 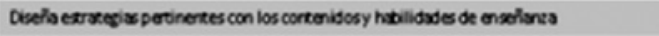 \\
\hline & & 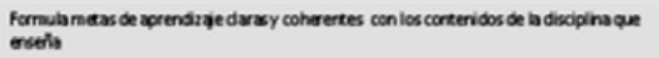 \\
\hline & & La plyriticacín incorporse el use de recurses disketiossrasiasos \\
\hline & \multirow{5}{*}{ 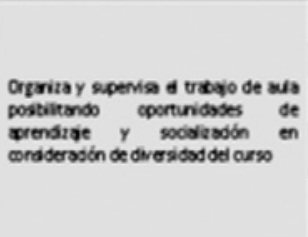 } & 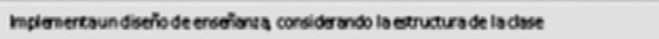 \\
\hline & & Ortritas ensdirsta en fundín des trajo persons del exudiste \\
\hline & & 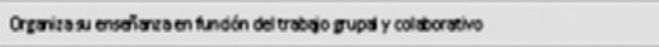 \\
\hline & & 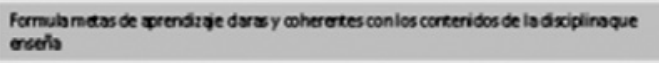 \\
\hline & & 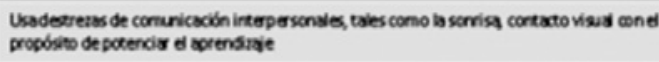 \\
\hline & \multirow{2}{*}{ 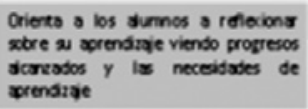 } & 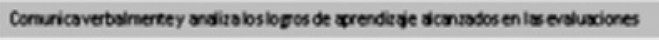 \\
\hline & & Evisenda los errores que presertan los estudates \\
\hline & \multirow{5}{*}{ 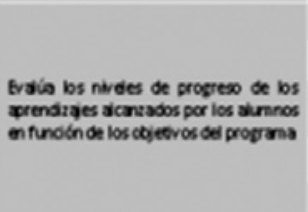 } & 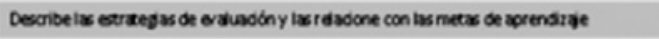 \\
\hline & & Conurica is neas de acrendragey los citerios de evaluaxion \\
\hline & & 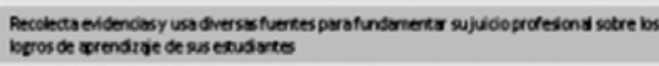 \\
\hline & & Usalos resultados dicareados paranodifica su enseliares \\
\hline & & nda postiescaripos de sccón pars entrentar ix dficitades de aprendiga \\
\hline
\end{tabular}

Para demostrar estas cuatro competencias, los estudiantes elaboraron un conjunto de evidencias en las que debieron reflexionar sobre "la preparación, la práctica y la experiencia", en relación a las competencias involucradas. Para ello, la base del portafolio la constituye el acopio de material concreto (guías, pruebas, planificaciones, ensayos académicos, artículos, etc.) en los que deben manifestarse competentes, a fin de registrar los episodios críticos presentados durante la práctica, ante los cuales levantan propuestas de solución, manifestando el espíritu investigativo que les permita fundamentar sus decisiones y reflexiones desde diversas fuentes (estudios, literatura especializada, monografías, entrevistas, encuestas, etc.). En correspondencia, el modelo didáctico tiene por sustento teórico las dimensiones filosóficas, sociológicas y pedagógicas que aporta del paradigma sociocrítico constructivista, que son la base para el fundamento teórico y metodológico en el que se fundamenta el modelo pedagógico concebido (Popkewitz I988; Habermas I966; Vygostky I985). 


\section{La génesis del modelo}

Para justificar la pertinencia de ofrecer un modelo de desarrollo de competencias, se refiere una descripción cualitativa de los resultados apreciados tras el diagnóstico, en el que se demostraron debilidades en los siguientes aspectos:

Manejo operativo y funcional de los Planes y Programas: lo que se manifestó en la incongruente selección de contenidos, planificaciones que no se estructuran de forma organizada en función de los requerimientos del programa, estilos de elaboración de tareas o actividades que no contemplan la diversidad y/o formas de evaluación convencionales o sin criterios consistentes.

Desarrollo de una metodología estructurada: comprometió la habilidad de algunos practicantes, para secuenciar la clase en tres períodos específicos, en lo que se ponían en juego la competencias asociadas al ajuste del tiempo (Soubal 2008), de manera que en la organización de la clase -tanto escrita como oral- tuviera cabida un inicio (donde se especificaran los objetivos, se consignara un título sugerente y se creara un conflicto cognitivo que permitiera contextualizar los objetivos de aprendizaje, en consideración de los intereses y características socioculturales de los estudiantes, y abriera la participación); un desarrollo (articulado alrededor de actividades de motivadores, en las que se utilice la variedad de recursos disponibles) y un cierre (con una efectiva recapitulación y contrastación entre los objetivos propuestos y el producto final). La conducción de la clase debiera mantener abiertas fórmulas de interacción que orienten al practicante respecto del nivel de comprensión con que los alumnos van siguiendo sus explicaciones y ofrecer así una retroalimentación apropiada al momento y lugar.

Competencias comunicativas en la interacción: lo que durante la observación de aula debió haberse mostrado en la habilidad para adecuar el discurso oral a los receptores, la capacidad de mantener la atención de los alumnos, la realización de desplazamientos y paneos visuales, un peso escénico que permitiera tener el control de 
los conflictos emergentes, liderazgo acorde a los requerimientos de un clima de aula propositivo y una actitud eficiente en conducir la clase a los objetivos planteados.

Competencias lingüísticas para dar cuenta de sus reflexiones: las diversas evidencias recogidas, manifestaron una incapacidad para describir con precisión ortográfica y/o coherencia conceptual, aquellos episodios críticos asociados a la praxis. Esto se manifestó también, en el diseño y redacción de situaciones de enseñanza que demostraran organización intencionada del trabajo de aula y la precisión léxica para elaborar por escrito propuestas de solución. Por su parte, el trabajo investigativo que requerían los diversos indicadores de desempeño, no siempre dio cuenta de una selección premeditada de entre una diversidad de fuentes teóricas y prácticas, con que fundamentar las decisiones metodológicas y didácticas del practicante. Las dificultades apreciadas en redactar con conciencia lingüística, se manifestaron en una estructura discursiva descuidada y una accidentada sintaxis.

Recursos didácticos: en un no despreciable contingente de estudiantes -ad portas de titularse-, se apreciaron recursos didácticos desgastados, inexistentes o convencionales. La inaplicación de los criterios de legibilidad (Alliende 2002) impidió evidenciar material de apoyo atractivo y novedoso. La naturaleza de las guías y pruebas elaboradas, dieron cuenta de dificultades para redactar instrucciones y preguntas coherentes con los objetivos, inconcordancias gramaticales, vicios léxicos, omisión de objetivos, selección de textos sin consideración de variables sociolingüísticas y, en general, propuestas de actividades poco pertinentes con las necesidades que imponen los diversos estilos de aprendizaje, ajustadas a las condiciones que imponen el tiempo y los recursos, asumiendo en su organización, que lo atractivo no es el contenido sino la actividad. La observación de aula, permitió apreciar un desaprovechamiento de los recursos didácticos generados de la síntesis. Esto, en general, permitió visualizar que los futuros profesores no asumen la importancia de construir material educativo para reforzar conocimientos, valores y actitudes, como el que constituye la estrategia de que los mismos alumnos letren la sala. De 
esta manera, se da uso didáctico a los diversos mapas conceptuales, afiches y esquemas con que se van sellando ciertos contenidos (Bruner 1995). Por otra parte, la organización física del mobiliario de la sala de clases, tendió al desarrollo de una clase de corte tradicional. Asimismo, el uso de TIC se limitó a la elaboración de power point de dudosa calidad: largos textos en lugar de conceptos, carencia de imágenes reforzadoras, y en lo técnico, sin la integración de botones de acción o hipervínculos que remitieran a fuentes que permitieran fortalecer los aprendizajes.

Lo anterior recoge las principales debilidades apreciadas, lo que demanda fortalecer competencias investigativas (asociadas al fundamento teórico que subyace tras la toma de decisiones), didácticas (para exhibir autonomía en la elaboración de material de apoyo, manejo de conocimientos fundamentales de evaluación al momento de crear pruebas, guías y uso de TIC) y comunicativas (dinámica de las interrelaciones internas y el contexto sociocultural de los discentes). En este sentido, las competencias comunicativas aportan significativamente a: "... abordar elementos de las dos esferas básicas de la personalidad, tanto la motivacional-afectiva como la cognitiva-instrumental” (Fernández González 1999, p. 42).

Estos resultados, dieron lugar a la implementación de un piloto (agosto a diciembre 20II) que buscó estimular el desarrollo de competencias didácticas, comunicativas e investigativas, para lo que proveyó de recursos y métodos. Se optó por aplicar el modelo a todos los estudiantes, a fin de no establecer odiosas diferencias entre los "mal evaluados de Práctica I" y el resto. No obstante, para efectos del análisis de los datos, se propone un seguimiento específico a los estudiantes mal evaluados, a fin de comparar su desempeño en su contexto natural y tras la implementación del piloto.

\section{Orientaciones para la aplicación del modelo}

De acuerdo a lo anterior y dados los requerimientos actuales del sistema escolar nacional, resulta perentorio que la práctica 
profesional sea concebida con la responsabilidad de ser el último espacio de formación que entrega el sistema educativo. Un espacio de formación disciplinar, personal y profesional, en que los futuros profesores: "[...] tengan acceso a oportunidades serias y sostenidas de aprendizaje”, sin las cuales sería: “... poco probable que lleguen a enseñar en términos que puedan alcanzar los exigentes estándares y menos que lleguen a participar de forma autónoma y crítica en la solución de los problemas educacionales, salvo que esto último, por cierto, sea sólo metáfora y discurso.” (Galaz et al. 2008, p. I34).

Por lo tanto, introducir un modelo que oriente el quehacer profesional, resulta coherente con los objetivos de alcanzar el desarrollo integral de los estudiantes. Esto se fortalece en el proceso que se desprende de la tarea de formar profesores, no sólo licenciados en educación. En consecuencia, el desafío conjunto es integrar al sistema educativo a profesionales que se validen como plenamente competentes, en el más amplio sentido del término. De esta manera, el problema científico de la investigación se define en la siguiente interrogante: ¿Cómo desarrollar las competencias profesionales necesarias para la enseñanza del castellano en la UCSH para mejorar la práctica profesional?

El modelo tiene como propósito proporcionar un marco conceptual, metodológico y estratégico, que favorezca el desarrollo de competencias en estudiantes en práctica profesional, por lo que el objeto de estudio de esta investigación, lo constituye la práctica profesional de castellano.

El campo de acción lo constituirán las competencias profesionales en castellano de estudiantes de $5^{\circ}$ año de la UCSH.

En virtud de las precisiones anteriores, se concibe como objetivo general de investigación, proponer un modelo didáctico que contribuya al desarrollo de competencias profesionales de los estudiantes en práctica profesional.

El modelo apuntó a desmarcarse de las prácticas tradicionales, dominadas por las metodologías propias de la transmisión, asumiendo 
una visión activa y democrática del conocimiento y de los procesos implicados en su construcción y transferencia, buscando así responder a los requerimientos de calidad y equidad que persigue la educación chilena. Lo anterior implicó realizar una serie de indagaciones teóricas y empíricas, como se aprecia, apoyadas en:

\section{I Indagaciones teóricas}

Histórico- lógico: Estudio del desarrollo curricular y los antecedentes históricos que permiten situar la concepción de competencias profesionales en el sistema educativo chileno.

Análisis documental: Selección de referentes que sitúan la pertinencia de la propuesta: las bases curriculares chilenas, el programa de castellano y otros documentos que regulan el sistema educativo en Chile.

Sistematización: El estudio teórico basado en los diferentes criterios epistemológicos, ontológicos y metodológicos y las experiencias acumuladas por la autora de la investigación permitieron la elaboración del modelo pedagógico.

Modelación: Relaciones esenciales en función de trazar aquellas competencias que identifican al profesor de castellano del sistema educativo chileno.

Método sistémico: El estudio y análisis de las relaciones esenciales permitió la orientación y elaboración del modelo propuesto.

Estadístico: Si bien el enfoque es predominantemente cualitativo, a través de gráfica y cuantificación, se objetivarán los hallazgos emanados de las encuestas y del 'acto docente'.

\subsection{Indagaciones empíricas}

Encuesta de percepción: Diagnóstico sobre el desarrollo actual de competencias asociadas a la enseñanza del Lenguaje y Comunicación en la escuela, de 54 estudiantes de castellano en proceso de práctica profesional. 
Recogida de evidencias: Observación del ejercicio docente auténtico y análisis de evidencias, para elaborar el modelo pedagógico propuesto, a objeto de responder a los principales problemas que plantea el desempeño de los estudiantes en práctica profesional, en la escuela. Evaluar el desempeño y detectar las debilidades presentadas en la praxis, para contribuir a reafirmar la necesidad de un modelo teórico de referencia.

Encuesta a supervisores: Para conocer los criterios en torno al modelo pedagógico.

Encuesta a egresados: Valorar el desempeño del "acto docente" y detectar las debilidades presentadas en la praxis. Ello permite contribuir a reafirmar la necesidad de contar con un modelo teórico de referencia.

Encuestas a autoridades de establecimientos educacionales: Para conocer su valoración con respecto al desarrollo de competencias profesionales del "acto docente" de estudiantes titulados en la $\mathrm{UCSH}$.

A pesar de utilizar procedimientos cuantitativos en la recogida de datos, se pone un mayor acento en los aspectos cualitativos, por lo que la interacción y retroalimentación devenida del proceso, permitirá reprogramar y evaluar las acciones. En concordancia con los métodos señalados, se movilizaron habilidades de pensamiento tales como: inducción-deducción, análisis-síntesis y reflexión crítica de las diversas etapas del proceso. 


\title{
5.3 El modelo pedagógico
}

Figura 2: El modelo

\section{MODELO DE DESARROLLO DE COMPETENCIAS PROFESIONALES}
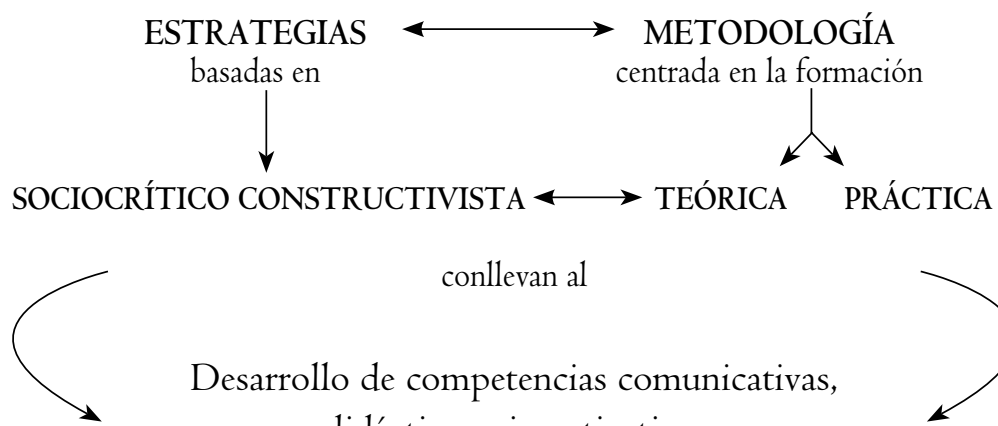

\author{
conllevan al \\ Desarrollo de competencias comunicativas, \\ didácticas e investigativas
}

La aplicación del modelo, exigió presentárselo al cuerpo de profesores supervisores, a fin de hacerles partícipes de la dinámica que implica y comprometerlos con su aplicación. ${ }^{5}$ Para ello se realizaron algunas reuniones divididas en sesiones, donde se explicaron los objetivos de la investigación, se presentó el modelo y se estructuró el trabajo que debiera surgir de este nuevo acompañamiento.

La primera sesión, busca recoger impresiones y propiciar la adherencia de los supervisores para la aplicación del modelo, puntualizándose en que se trata de orientaciones metodológicas que se presentarán en las primeras reuniones, que tienen por objetivo establecer procedimientos que se irán verificando y afianzando conforme avance el semestre. De esta manera, se explica el contexto de la investigación y sus objetivos, se expone el cuadro sinóptico de desempeño y se exhiben evidencias auténticas, que justifican la pertinencia de la intervención. Al mismo tiempo, se expone el análisis cualitativo del análisis de los bajos desempeños, tras lo cual se presenta el modelo pedagógico y los referentes teóricos en los que se enmarca.

5 Se trata de tres profesores de dilatada trayectoria profesional. 
Expuestos los fundamentos que justifican la pertinencia de re-orientar el acompañamiento, en las sesiones siguientes, se dan las orientaciones metodológico-didácticas.

\section{La aplicación del modelo}

La sugerencia de adoptar/adaptar un nuevo estilo de conducción de reuniones de supervisión, busca cautelar que ésta no sea una zona de catarsis colectiva, sino un espacio de aprendizaje, que contribuya a nivelar los variados aspectos disciplinares, comunicativos, metodológicos, administrativos, didácticos, etc. involucrados en el proceso - con especial énfasis en los propios del currículum del sector de Lengua Castellana y Comunicación- y no se suponga tácitamente, que éstos fueron adquiridos por los practicantes, por el solo hecho de haber aprobado los diversos cursos de formación.

Se sugiere adaptar libremente los temas de cada reunión, con énfasis en conducirla orientando el desarrollo de:

Competencias orales y comunicativas: verificables en las diversas etapas que plantean las relatorías que deberán realizar.

Manejo conceptual: mediante la distribución de tareas específicas, de dificultad progresiva, que fortalezcan los conocimientos propios de la disciplina y los variados aspectos curriculares de la profesión.

Capacidad de investigar autónomamente: los profesoressupervisores propondrán temas y tópicos, en concordancia con el objetivo de desarrollar en los practicantes, habilidades relacionadas con la selección de información, recurriendo a diversas fuentes, de manera que la investigación sea la base sobre la cual fundamente sus decisiones y propuestas de solución, conforme vayan surgiendo episodios críticos en su praxis.

Afianzamiento metodológico-didáctico: competencias asociadas al saber enseñar la disciplina, verificables en la calidad y coherencia de 
las planificaciones, el estilo de enseñanza, los criterios de evaluación y, transversalmente, en la reflexión sobre el acto pedagógico.

Estos temas deben surgir de lo que el profesor-colaborador aprecie deficitario, por lo que en esta instancia, el acompañamiento debe dar respuesta a nivelar vacíos conceptuales y desarrollar competencias comunicativas y sociales, acordes a las progresivas exigencias que imponga el proceso.

Lo anterior provee las bases para conducir la fase de reflexión acerca de la importancia de desarrollar tanto conocimientos fundamentales de la disciplina, como la apropiación de herramientas metodológicas que les permitan fundamentar sus reflexiones sobre la práctica. En esta misma sesión, se exponen las primeras orientaciones metodológicodidácticas, que versan sobre los siguientes tópicos:

\section{I El uso racional de TIC}

Se debe prestar especial atención a que el uso de TIC se asuma como un medio que facilita el aprendizaje, por lo que se deben desarrollar competencias de forma y de fondo, en consecuencia con el paradigma de la sociedad del conocimiento (Castells I997; Labra et al. 2008; Marcelo 200I; Toffler 1995). Esto justifica la pertinencia de las orientaciones que propone el modelo, a fin de importar la didáctica y el tratamiento de la tecnología, de manera de hacerse cargo del perfil multimedial del estudiante de hoy. ${ }^{6}$

La visión sistémica de los enfoques pedagógicos fundados en el constructivismo, señalan la pertinencia de elicitar procesos de aprendizaje basados en el procesamiento de la información, a fin de evidenciar desarrollos metacognitivos consistentes. El aludido uso racional de TIC, considera que la información se transforma y utiliza, no es un dato.

6 A través de fichas didácticas, pautas de evaluación y rúbricas, se presentan uno a uno los temas que el modelo desarrolla. El espacio no permite más que hacer una breve descripción de estos tópicos. 
Así, el acompañamiento en el uso racional de TIC, debiera conducir a que los profesores-alumnos desarrollen habilidades para elaborar material audiovisual estructurado, pertinente y significativo. Lo anterior implica demostrar competencias, tanto para la búsqueda y selección de recursos para el aprendizaje en la red, como para el manejo de plataformas virtuales, que constituyan otro medio para comunicarse con sus profesores y alumnos.

Por tanto, el uso de recursos tecnológicos no debe reducirse al mero uso de power point, sino a la utilización de las tecnologías que permitan la interconexión y atención a universos amplios de individuos, en concordancia con las formas de relación que imponen las escuelas y el mundo globalizado. Lo anterior posibilitaría el desarrollo de competencias asociadas a lograr la integración de las TIC en el ejercicio docente, así como en lo relacionado a la investigación y diseño de recursos educativos basados en las potencialidades de Internet.

En paralelo, ha de considerarse guiar el fortalecimiento de las competencias orales y comunicativas de los estudiantes. Para ello, el modelo propone la utilización de un sencillo formato, adaptado por la investigadora, que busca estimular el desarrollo del conjunto de habilidades involucradas en la capacidad de exponer con fluidez, seguridad, respeto del tiempo, y a la vez, mostrarse sólida, tanto en los fundamentos disciplinares de lo que expone (saber sabio), como en la habilidad para comunicarlo eficazmente (saber enseñado). Lo anterior implica propiciar el desarrollo de variadas competencias comunicativas, a fin de dar respuesta a los requerimientos dinámicos de la interacción áulica (Ruiz Socarrás I994; Chevallard 1997).

\subsection{La Relatoría}

Integrar el formato relatorías como modelo de presentación oral, busca proporcionar una guía concreta a través de un sencillo esquema, de los aspectos funcionales, estructurales y pedagógicos que ayudarían a incrementar competencias comunicativas y orales. De esta manera, se propone una estructura que contextualiza el tema, presenta los contenidos y objetivos, y busca mostrarse eficiente y desarrollarse 
en coherencia con la situación de comunicación. Asimismo, da el escenario para exhibir competencias en el ajuste del tiempo, así como las relacionadas con actividades cognitivas que permitan elaborar ejemplos propios y pertinentes a los objetivos de aprendizaje para exponerlos ante la audiencia, y que se hace cargo de uno de los aspectos, en general, más deprimidos: culminar la exposición con un cierre intencionado y programado, que recapitule las ideas fuerza.

La consecución de las competencias relacionadas con estructurar temática y comunicativamente una clase, ha de ser apoyada durante todo el proceso de formación final, lo que requiere que los profesores supervisores soliciten exponer una diversidad de temas asociados al quehacer pedagógico. Para la consecución de estos fines, el modelo provee una pauta específica.

\subsection{El esqueleto de Clases}

En este punto, el modelo define elementos estructurales específicos, que debieran constituirse en lo que se ha convenido en llamar el esqueleto de la clase, que debiera observarse en cada intervención de aula. Tres son sus ejes articuladores:

Inicio: la puesta en escena

I. Escribir la fecha, título de la clase (procurando ser originales y creativos) y objetivos de aprendizaje. La redacción de los objetivos debe adaptarse de lo señalado como Aprendizajes Esperados para el curso o nivel; no redactarse textual. Se recomienda el uso de plumones de colores contrastantes, para subrayar ideas y/ jerarquizar elementos o conceptos.

2. Activación de los conocimientos previos. Quién sabe qué del tema, dónde lo escuchó o vio.

3. Contextualización de la temática, estimulando la asociación entre lo que aprenderán (objetivos) y la vida cotidiana (para qué servirá conocer qué). 
4. Quiebre cognitivo: utilizar variados recursos para despertar la atención e interés de los receptores. Pueden ser orales, escritos o audiovisuales.

5. Llegada inicial: exposición clara y dinámica; lenguaje adecuado a los receptores, que tienda al incremento del vocabulario. Desplazarse por el aula, hacer paneos visuales, demostrar peso escénico.

6. Clima de aula: promover normas de cortesía, respeto de turnos, mantener el control de la situación. Manejar conflictos, negociar, atender a necesidades emergentes.

Desarrollo: aspectos metodológicos

I. Exposición de conceptos: claridad y fluidez discursiva, pertinencia con el nivel sociocultural de los receptores. Exhibir capacidad para mantener la atención de la audiencia. Utilización de recursos didácticos de diverso tipo. Uso intencionado y participativo del recurso pizarrón: orden en la exposición de lo que se escriba en él, tamaño de letra visible desde los últimos asientos, integración de esquemas y/o mapas conceptuales afines, para ilustrar o sintetizar conceptos o ideas fuerza.

\section{Actividades:}

Crear actividades contextualizadas, concordantes con las habilidades que se busca desarrollar: lectura, escritura, oralidad.

Manejar una diversidad de formas de organizar la interacción: trabajos individuales o grupales, exposición ante pares, etc., considerando los diversos estilos de aprendizaje presentes en el aula (Alonso et al. 1994).

Organizar eficientemente el tiempo, señalando con precisión los requeridos para la realización de cada actividad.

Monitorear avances y dificultades. 
Triangular coherentemente: Objetivos/ Actividades/ Evaluación.

Hacerlos participar y cautelar celosamente que se escuchen y respeten.

Reforzar positivamente cualquier participación.

Dar el mejor esfuerzo por elaborar actividades creativas e innovadoras, ajustadas al contexto.

Término: cierre de la clase

I. Recapitulación en forma, de los contenidos de la exposición: interrogar al curso ¿qué aprendimos hoy?, y/o comparar el objetivo con lo que hicieron. En este contexto no importa que se provoque cierto caos; privilegiar las intervenciones.

2. Recoger información respecto de los objetivos propuestos.

3. Favorecer la auto y co-evaluación.

4. Utilizar algún recurso de cierre oral y colaborativo. (Esquemas u organizadores gráficos que los alumnos alcancen a copiar.) Emplear didácticamente el pizarrón al mediar la síntesis.

5. Demostrar competencia en el ajuste del tiempo, de manera de cumplir con los tres ejes propuestos: Inicio/ Desarrollo/ Cierre.

6. Utilizar los cuadros-síntesis elaborados en conjunto, como objetos de aprendizaje.

\subsection{Las Guías de Apoyo}

Por otra parte, un elemento fundamental del trabajo docente, es preparar guías y material de apoyo, cuya finalidad es complementar, reforzar o ampliar aprendizajes. Su elaboración ha de ser cuidadosa e intencionada. Hace, por tanto, necesaria una didáctica específica. El material elaborado por el profesor practicante, debiera considerar dos dimensiones: el aspecto formal y la naturaleza de las actividades 
que plantea, dimensiones desde las que se establece como su propuesta didáctica. Esta 'doble dimensionalidad' del material de apoyo, se resume específicamente en una ficha didáctica, sin embargo, describiremos algunas consideraciones fundamentales:

I. Lo formal: las competencias de los futuros profesores, en este ámbito, se verifican en la organización gráfica, la propiedad gramatical, la corrección ortográfica, la coherencia global, lineal y pragmática del material preparado, así como en su cohesión léxica. Esto compromete, además, las habilidades del futuro profesor, para adecuarse creativamente a las particularidades del contexto. Los criterios de legibilidad resultan de gran valor para resaltar dos elementos vitales de la acción pedagógica: la selección cuidadosa de las lecturas y textos empleados (en y fuera del aula), como la producción de material de apoyo, que no sólo dé importancia a la legibilidad material y estructural (tamaño, fuente, diagramación intencionada, superestructura), sino a la legibilidad lingüística, conceptual, psicológica y pragmática, de manera que el producto didáctico final estimule -efectivamente- la consecución de los objetivos de aprendizaje para el contexto que fueron creados.

2. La naturaleza de las actividades: un punto no menor, son las actividades emanadas de la propuesta didáctica. Su contenido, procedimientos y estrategias deben propiciar el desarrollo de diversos tipos de aprendizajes, dentro y fuera de la sala (Román Pérez y Díez López 199I), en concordancia con los diversos dominios solicitados por el currículum vigente.

La coordinación y conducción de la clase deben permitir el desarrollo de las actividades propuestas en el material de apoyo (guías, módulos didácticos, etc.), lo que compromete las competencias del profesor practicante, de crear oportunidades para que los receptores, construyan, presenten y evalúen su propio conocimiento. Así, las actividades diseñadas por el futuro profesor, debieran permitir verificar competencias para la organización del trabajo en tiempos específicos y manejar situaciones emergentes: circulación de los 
alumnos, indisciplina, interrupciones, etc., de manera de cautelar que se produzca la metacognición.

Se sugiere considerar las posibilidades de aprendizaje que otorgan las actividades de resolución colaborativas. Sin embargo, para obtener los beneficios de esta modalidad, el profesor practicante deberá hacer notas las diferencias de trabajar en equipo y trabajar en grupo. En este sentido, el futuro docente debe organizar la interacción, procurando dejar en cada grupo de trabajo a un alumno aventajado, en cuya actividad modelice a los otros y se fortalezca él mismo.

\subsection{Las evaluaciones escritas}

La instancia de la evaluación es fundamental para evidenciar competencias asociadas al saber enseñar la disciplina. Para ello, el futuro profesor debe conocer y manejar diversos enfoques, procedimientos e instrumentos evaluativos. Uno de éstos, lo constituyen las pruebas escritas.

Nos centraremos en la evaluación calificada porque, como parte del proceso de enseñanza, se erige como una herramienta que permite el control y potencia el aprendizaje. Cabe señalar que el profesor practicante debiera demostrar competencias para conocer las características y alcances de los diversos tipos de instrumentos de medición nacionales e internacionales, en los que nuestro país está involucrado.

Los instrumentos permiten diferentes modos de formular preguntas. Así, algunas preguntas permitirán evaluar la memorización de información (preguntas de respuesta guiada, verdadero o falso, términos pareados, definición de conceptos), mientras que otras, objetivar el manejo de destrezas cognitivas más complejas (de desarrollo, interpretativas, valorativas o argumentativas, y de resolución de problemas).

Un profesor competente no debiera relegar este importante proceso al azar: sus decisiones y material debieran tender a desarrollar habilidades de complejidad creciente. 
- Aspectos formales:

I. Aplicar las orientaciones dadas para las Guías (En el punto 6.4).

2. La formulación de las preguntas debe incluir los siguientes elementos:

- Un verbo para indicar lo que el estudiante ha de ser capaz de realizar.

- Oraciones cortas y precisas. Evitar la ambigüedad.

- Considerar integrar preguntas abiertas y cerradas.

- Las preguntas de desarrollo deben ser redactadas de manera que se distinga claramente el nivel de detalle que persigue, así como el carácter o contexto de la ejecución.

- Aspectos metodológico-didácticos:

I. Al asignar puntajes, contemplar que las actividades cognitivas emanadas de las preguntas formuladas, considere al menos tres niveles de desempeño: inicial, intermedio y avanzado.

2. La evaluación de la redacción y la ortografía debiera escindirse de los requerimientos de reflexión y desarrollo creativo. Por tanto, cualquier tipo de descuento por este concepto, debiera considerar, previamente, el tipo de competencias genéricas perseguidas.

3. Los enunciados debieran ser lo suficientemente llanos, de manera que faciliten la comprobación del grado de adquisición de aprendizajes (Luna, 2004).

4. Los instrumentos han de ser variados y contemplar el desarrollo tanto de lo oral como lo escrito.

5. Para evaluar instancias de aprendizaje orales, se darán a conocer los criterios de evaluación antes de la ejecución de la tarea, y tales criterios se objetivarán mediante pautas, rúbricas, tablas de cotejo, etc., que especifiquen claramente los niveles de desempeño perseguidos.

A través de la evaluación, el docente reflexiona sobre su práctica y su propio desempeño, y pone en juego su capacidad para autoevaluar las estrategias y materiales implementados para alcanzar resultados de aprendizaje, según el carácter y nivel de los contenidos enseñados (Escalona Orcao y Loscertales Palomar 2007). 
Deben considerarse una variedad de formatos de evaluación (tipo PSU, tipo SIMCE, selección múltiple, verdadero y falso, términos pareados, etc.) que ofrecen la posibilidad de responder específicamente (sabe o no sabe: preguntas cerradas), aunque integrar especialmente preguntas abiertas (de desarrollo) más apropiadas para evidenciar habilidades de nivel superior, como las relacionadas con emitir opiniones, fundamentar o formular juicios crítico-valorativos.

Este aspecto justifica nuestra insistencia en señalar que no se consideren las habilidades ortográficas, en subordinación de las competencias relacionadas con desarrollar productores de textos. No se trata de relegar la importancia de que la producción textual se ajuste a la norma, sin embargo, desde un punto de vista comunicativo y funcional, se ha de considerar el valor de las producciones textuales como fenómeno discursivo, cognitivo, social y cultural. (Jolibert 1997).

De los resultados de aprendizaje logrados por los alumnos, el docente competente asumirá la urgencia de re-orientar el proceso de enseñanza-aprendizaje elicitado, como consecuencia de dicha evaluación. Esto permitirá tomar decisiones, mejorar el estilo de enseñanza y desarrollarse como profesional.

\subsection{La Planificación}

La planificación competente exige conocimiento profundo de aspectos curriculares. Independientemente de sus modelos (academicista, conductista, constructivista o cognitivo), el futuro profesor debiera comprender los enfoques, conceptos principales, objetivos fundamentales y contenidos mínimos que sustentan los ejes del currículum en el sector de la especialidad, y tener una postura analítica frente a ellos. Pero, además, debe estar familiarizado y ser capaz de usar los diversos instrumentos curriculares que provee el sistema educativo chileno, tales como las Bases Curriculares, los Programas de Estudio, los Mapas de Progreso, los Niveles de Logro SIMCE, así como conocimiento de los textos escolares, relacionados con los enfoques de la disciplina que asumen diversas editoriales. 
La planificación constituye la evidencia tangible tanto del grado de dominio del docente de los objetivos perseguidos en los Planes y Programas del sector, como del conocimiento de las características socioculturales del curso y de sus habilidades para asociar contenidos a actividades pertinentes y significativas, con lo que evidencia sus competencias pedagógicas.

La postura metodológica, la naturaleza de las decisiones (u omisiones) y el conjunto de recursos didácticos con que proyecta una clase, son actividades que manifiestan una posición ideológica frente a la labor docente, o sea, revela el sustrato de su discurso pedagógico.

En esta instancia, el profesor debe tener la flexibilidad de adaptarse a la estructura sugerida por la institución que la acoge, pero en independencia de su forma, la limpia ejecución de esta competencia manifiesta, en su realización, que conoce y maneja los diversos contenidos y que los relaciona con metas coherentes, contextualizadas y progresivas de aprendizaje, donde se propicia tanto el desarrollo de habilidades de pensamiento (interpretar, analizar, inferir, explicar, autorregular), como la construcción de conocimientos conceptuales, actitudinales y procedimentales. Y dada la libertad de los diversos proyectos educativos para hacer sus propias exigencias respecto del tipo de planificaciones en que enmarcan el trabajo educativo, el futuro profesor, debiera exhibir conocimiento de las diversas formas de planificar. Con todo, las más utilizadas actualmente son las de trayecto, por tanto en éstas se centran nuestras orientaciones.

Aprendizajes Esperados: También denominados objetivos fundamentales o generales. Se redactan literalmente de los Planes y Programas, sin omisiones. (Reconocen..., Describen..., Relacionan..., etc.)

- Contenidos: Llamados también contenidos programáticos u objetivos especificos. Se refiere al tema o tópico a abordar. Su selección debe ser coherente con el aprendizaje esperado.

- Actividades de Aprendizaje: (o actividades sugeridas). Este apartado debe consignar claramente los tres momentos de la clase: Inicio 
/Desarrollo/ Final. La redacción debe dar cuenta de lo que hace el alumno, no el docente.

En el inicio, integrar las orientaciones metodológico-didácticas, previstas para el "esqueleto de la clase".

- Capacidades/Destrezas: Lo que se espera afianzar y desarrollar. Involucran actividades cognitivas.

- Valores/ Actitudes (u objetivos transversales): Enmarca lo que debiera ser la actitud, responsabilidad, etc. La propuesta didáctica debe hacerse cargo de dar cuenta del compromiso "declarado", a fin de recogerlo.

- Recursos: Especificar si se utilizará material concreto, audiovisual, etc.

- Evaluación: La clase siempre es evaluada (evaluación de proceso, formativa, sumativa, global, etc.) y los instrumentos para evidenciarla, variados: autoevaluación, co-evaluación, heteroevaluación.

Como se ve, la planificación, organiza el diseño y preparación de la secuencia didáctica requerida para hacer enseñables los contenidos seleccionados. En esta fase, el profesor tiene amplia libertad para seleccionar objetivos fundamentales y aprendizajes esperados del currículum.

Tras la selección, se diseñan las estrategias y métodos más adecuados para lograr los aprendizajes proyectados, se plantean y preparan las estrategias de evaluación (antes, durante y/o después del proceso), se elaboran o adaptan los recursos de aprendizaje que se utilizarán, y se define la interacción que se quiere lograr con los alumnos, lo que implica una cuidadosa organización de los tiempos involucrados para los logros finales.

Finalmente, el modelo provee recomendaciones para afianzar la composición escrita y ortografía, elaborándose las orientaciones en virtud de la importancia que le asigna el currículum a adquirir competencias de producción escrita. 
Así, evidenciar competencias lectoescritoras, implica-necesariamentecomprender las formas lingüísticas y la estructura genérica del texto, de manera de adecuar los recursos que se posean, para la presentación de la información, dependiendo de la situación y objetivos de comunicación.

Las orientaciones del modelo se elaboran tras el análisis cuantitativo y cualitativo de los textos producidos por los profesores practicantes, manifiestos en el conjunto y variedad de evidencias con que buscaban objetivar sus conocimientos, compartir las reflexiones emanadas del proceso educativo, ofrecer solución a episodios críticos, etc., y hacerlo en correspondencia y respeto por las competencias que imponen la forma y fondo.

Respecto del fondo, se observó una marcada debilidad para establecer relaciones entre los conceptos, asignar sentido a la información recibida y problematizar acerca del Acto Docente. La carencia de lecturas previas, se expresó en productos finales superficiales, que no se remitían a fuentes para complementar, ilustrar o exponer los puntos de vista que buscaban defender en tales comunicaciones.

Los problemas de forma, dificultan la tarea de redactar ensayos con propiedad, utilizar variados nexos y conectores para expresar pensamientos e ideas, manejar un léxico acorde a la disciplina que dé efectiva cuenta de estar frente a un profesional competente, esto es, con sólidas competencias gramaticales, pragmáticas y comunicativas, capaz de transformar el conocimiento.

En este contexto, el conjunto de evidencias del diagnóstico, permitió constatar una generalizada debilidad ante los requerimientos de redactar tanto con coherencia argumentativa, como con precisión y corrección gramatical. Y por ser una de las responsabilidades de los futuros profesores especialistas implementar una didáctica de producción textual, si evidencian deprimidas sus propias habilidades para elaborar textos, será poco probable que logren desarrollar, competentemente, tales requerimientos en sus alumnos (Van Dijk \& Kinstch 1983, Cassany I990). 
Así las cosas, las orientaciones que entrega este modelo, asumen que la propia redacción es lo primero que el futuro docente debe resolver, por ser la base de su eventual ejercicio reflexivo crítico y capacidad investigadora. No olvidemos que el manejo competente del código escrito compromete tanto conocimientos gramaticales (fonética, morfología, sintaxis y léxico) como el conjunto de habilidades relacionadas con la adecuación (variedad y registro pertinente), coherencia (seleccionar la información y organizar la estructura comunicativa de una manera determinada) y cohesión (recursos gramaticales y referencias al propio texto que se encadenan, oración por oración) relacionados en el proceso de comunicación.

En consecuencia, las orientaciones que provee el modelo para abordar el proceso de composición escrita, así como un apartado específico de ortografía, no se desarrollan en este artículo por razones de espacio, aunque debemos señalar que dichas orientaciones sugieren el desarrollo de la expresión lingüística del pensamiento, en ajuste irrestricto a las convenciones gramaticales, discursivas y textuales. Las orientaciones del modelo, en este punto, enmarcan los tópicos disciplinares que todo futuro docente debiera saber y manejar, y a través de descripciones breves, pretenden instalar la necesidad de que sea el propio docente el que acoja el llamado a investigar autónomamente, a fin de reforzar/ nivelar/ descubrir aquellos aspectos del manejo del código lingüístico en los que se reconozca débil.

\section{Consideraciones finales}

La educación, como uno de los ejes esenciales de la sociedad, ha sido fundamental para favorecer que los sujetos se desarrollen e integren plenamente en el contexto sociocultural en que se desenvuelven. Impone, por tanto, una demanda a las instituciones de educación superior responsables de impartir pedagogía, que no puede satisfacerse por completo, a menos que se desarrolle integralmente a sus principales agentes: los profesores.

Le corresponde así, al sistema educativo, hacerse cargo de entregar herramientas para que sus futuros profesores, cuenten con variados 
recursos para transferir sus saberes conceptuales, en la práctica, de manera de generar aprendizajes significativos, trascendentales y útiles para toda la vida de sus educandos. Para ello, se hace necesario revisar y actualizar constantemente la metodología y didáctica empleadas en la práctica pedagógica cotidiana.

Lo anterior proveyó el marco necesario que le permitió a la autora concebir su propuesta y que, como tal, forma parte de una investigación en pleno curso. Este modelo pedagógico se elabora a partir de un sustento teórico que abarca las dimensiones filosóficas, sociológicas, lingüísticas y pedagógicas, que son la base para el fundamento metodológico-didáctico en que sitúa su visión holística del problema.

Tras su aplicación, busca probarse como un recurso pedagógico que potencie el desarrollo de competencias, de manera de permitirle al profesor de aula, transposicionar el saber conceptual en saber práctico. De esta manera, proyecta apoyar el proceso de formación docente.

Se tienen como base, los instrumentos "portafolios virtual" y "observación de aula", a fin de cuantificar el desempeño docente en la pre y post evaluación, en un período de dos años.

Las herramientas que se proveen, fundadas en una mirada socioconstructivista de la enseñanza, buscan orientar la relación y la práctica pedagógica, en función a la puesta en escena áulica y la toma de decisiones, a fin de sistematizar una práctica pedagógica que se certifique como competente. Sólo nos resta verificar la validez de este modelo, lo que sin dudas, será tema para un siguiente artículo.

\section{Bibliografía}

Aebli, H. (200I). Factores de la enseñanza que favorecen el aprendizaje autónomo. Madrid: Narcea, S. A. de Ediciones.

Albornoz, M. (200I). Política Científica y tecnológica: Una visión desde América Latina. En Revista CTS+I. No I. Disponible en: http://www.campus-oei.org/ 
revistactsi/numeroI/albornoz.htm

Aldape, T. (2008). Desarrollo de Las Competencias del Docente. Demanda de la Aldea Global Siglo XXI. Editorial LibrosEnRed.

Alliende, F. (2002). La legibilidad de los textos. Santiago de Chile: Ed. Andrés Bello.

Alonso, C.; Gallego, D. \& Honey, P. (1994). Los Estilos de Aprendizaje. Procedimientos

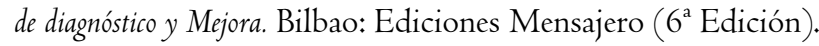

Álvarez de Zayas, C. (1999). La escuela en la vida. La Habana: Edit. Félix Varela. C. $3^{\text {a }}$ Edic.

Bruner, J. (I995). Desarrollo Cognitivo y Educación. Madrid: Ediciones Morata S. L.

Cassany, D. (1990). "Enfoques didácticos para la enseñanza de la expresión escrita”. En: Comunicación, Lenguaje y Educación, n 6, pp. 63-8I.

Castells, M. (1997). “La era de la información”. Economía, sociedad y cultura. 3 vols. España: Alianza Editorial.

Cuaderno de trabajo de práctica profesional. Escuela de Educación en Humanidades y Ciencias. (20I I). Santiago de Chile: Universidad Católica Silva Henríquez. p. 6.

Chevallard, Y. (1997). La transposición didáctica. Del Saber Sabio al Saber enseñado. Buenos Aires: Aiqué.

Escalona Orcao, A.I. y Loscertales Palomar, B. (2007). V Jornadas de Investigación en Docencia Universitaria. Pautas y materiales para la enseñanza y el aprendizaje de competencias de comunicación en los nuevos títulos de Grado. Alicante [Documento en PDF]: http://www.eduonline.ua.es/jornadas2007/ comunicaciones/2F8.pdf

Fernández González, A. M. (1999). La competencia comunicativa del docente: Exigencia para una práctica pedagógica interactiva con profesionalismo. La Habana.

Frade, L. (2006). Desarrollo de competencias en educación: desde preescolar basta bacbillerato. Biblioteca para directivos y supervisores escolares en el D.F. México: SEP.

Galaz, A.; Noguera, M.I. \& Urrutia, E. (2008). Revista Foro Educacional, P. I34. UCSH.

Gimeno Sacristán, J. (2008). Educar por competencias. ¿Qué bay de nuevo? (Comp.) Madrid: Morata.

Habermas, J. (1966). Teoría y Práctica: ensayos de filosofía social. Buenos Aires: Editorial Sur.

Halliday, M. A. K \& Martin, J, R. (1993). Writing Science. Literacy and Discursive Power. London: University of Pittsburgh Press.

Jolibert, J. (1997). "Libros: ofrecer contactos múltiples y diversificados”. En: Formar niños lectores de textos. Santiago de Chile: Dolmen. $7^{\text {a }}$ Edic. pp. 
La práctica profesional como problema de aprendizaje: orientaciones para la aplicación de un modelo de desarrollo de competencias - Marisa Guzmán Munita

\section{II7-134.}

Labra, J. y otros (2008). Informe: Integración de las TIC en la Formación Inicial Docente. Universidad de Santiago de Chile. UMCE, entidad asociada

Luna, J. A. (2004). Un manual de aprendizaje reflexivo y vivencial: Teoría y Práctica. USA: RoutledgerFalmer.

Marcelo García, C. (200I). "La educación ante la sociedad del conocimiento". En: Revista complutense de educación. Vol. I2, nº 2, pp. 53I-593.

Martínez Quijano, M. (2008). "El tutor pedagógico en la formación docente”. Revista Científico-Metodológica. Universidad de Ciencias Pedagógicas "Enrique José Varona". № 46, enero-junio.

Ministerio de Educación (2009). Fundamentación del Ajuste Curricular en el Sector de Lenguaje y Comunicación. Unidad de Currículum y Evaluación. $2^{a}$ Edición.

Modelo de Formación de la Universidad Católica Cardenal Raúl Silva Henríquez. (Documento de circulación interna). Septiembre de 2003, p. II.

Noguera, M. I. et al. El uso del portafolio en la formación práctica. Proyecto MECESUP Prácticas (2009- 2010). Universidad Católica Silva Henríquez.

Popkewitz, T.S. (1988). Paradigma e ideología en la investigación educativa. Madrid: Mondadori.

Pérez Gómez, A. (2007). Más allá del academicismo: los desafíos de la escuela en la era de la información y la perplejidad. Universidad de Málaga.

Román Pérez, M. y Díez López, E. (I99I). Currículo y Aprendizaje. Navarra: Dirección Provincial del MEC. Mapas de Progreso del Aprendizaje. Sector Lenguaje y Comunicación.

Ruiz Socarrás, J. M. (1994). "Los métodos de enseñanza en la Educación Superior”. En: Revista Cubana de Educación Superior. Vol. 2, n I4, pp. I2I-I24.

Soubal, S. (2008). "La gestión del aprendizaje. Algunas preguntas y respuestas en relación con el desarrollo del pensamiento en los estudiantes”. En: Polis, Revista de la Universidad Bolivariana. Nº 7, Vol. 2I, pp. 3 I I-337. [Documento en línea] http://redalyc.uaemex.mx/pdf/305/30502I I5. pdf

Toffler, A. (1995). El cambio de poder. Barcelona: Plaza Janés.

Van Dijk, T. \& Kintsch, W. (1983). Strategies of discourse comprehension. New York: Academic Press.

Verret. M. (1975). Le temps des études. París: Honoré-Champion.

Vygotsky, L. (1985). Pensamiento y Lenguaje. Buenos Aires: Pléyade. 